\title{
RGB BASED SENSOR FOR SEMI-SPHERICAL LIGHT MEASUREMENTS
}

\author{
Peteris Apse-Apsitis, Ansis Avotins, Jolanta Graudone, Ricards Porins \\ Riga Technical University, Latvia \\ peteris.apse-apsitis@rtu.lv, ansis.avotins@rtu.lv
}

\begin{abstract}
Different light sources emit light in various directions in a different spectrum, making this a complex task in real application environments. In the case of a greenhouse, evaluation of spherical lighting must be carried out to understand how much power the plant is getting at the top, inter, and bottom layers. Part of the light is both absorbed and reflected by plant leaves, and the greenhouse elements themselves. Determination of received $\mu$ mol amount from sunlight and/or artificial lighting is vital for plant growth and also the energy efficiency of the total greenhouse system. For best results, this parameter needs measurements over time by a specific sensor for this application. The article deals with a new design of the RGB light sensor - RGB sensor matrix in spherical form, where obtained raw data are processed on the controller and through communication network sent to the IoT database. The article deals with the initial measurement design, and tests for the proof of the concept and later overall discussion. The conclusion at the end is that such an approach can be applied for light parameter monitoring in greenhouse systems.
\end{abstract}

Keywords: RGB spectrum, light measurement, IoT.

\section{Introduction}

The demand for fresh vegetables and healthy food is increasing, thus also many industrial greenhouses, vertical farming, hydroponics and other growing facilities emerge more and more frequently. The tendency in greenhouse automation also asks for more control/sensor options, such as quality parameter measurements for feedback, in order to control greenhouse heating, ventilation, climate equipment in more precise and energy-efficient way. Nowadays systems with Internet of Things (IoT) become more and more popular for such applications.

The article deals with a lighting measurement system that is able to count micromoles from semispherical directions, thus in a greenhouse it is possible to detect light amount that hits the sensor from top, from sides and reflected light from the floor. The proper lighting system management can save a lot of electrical energy, due to fact that greenhouse lighting system is the main electrical energy consumer. This is due to the fact, that greenhouse has top lighting, typically High-Pressure Sodium vapour lamps (400W-600W) or equivalent LED lamps (120-200W), placed at the ceiling level in single array. Then there are also interlighting systems, having one, two or even three LED lamps in one array, that are placed in the crop level, to have more light (micromoles) in the leaf and fruit (tomatoes, cucumber, etc). These artificial lights are switched ON or OFF, depending on sunlight availability during the day or according to specific algorithms used by the greenhouse system or managing person. Currently for micromole measurements, relatively expensive quantum based sensors, like Li-COR LI-190R [1] are used to measure photosynthetically active radiation (PAR) values, typically placing single sensor in the greenhouse centre, controlling the whole greenhouse system ( 0.5 hectares). If the greenhouse is growing different varieties of tomatoes, and under different artificial (mixed) lighting systems, then the feedback value from such lighting mix will be different in each place/height, as tomato leaves are growing very differently in each sector, having various leaf coverage area, etc. SI-NDVI sensors [2] would be introduced into evaluation and greenhouse system control algorithms, as they have potential to monitor plant water stress detection in greenhouses [3], according to [4], spectral reflectance by vegetation in ranges of $0.4 \mu \mathrm{m}$ to $2.5 \mu \mathrm{m}$ can indicate healthy, stressed or severely stressed vegetation. From [5] a single image NDVI (SI-NDVI) gives a new way to derive spectral character from a single RGB image, thus it can be applied to indoor greenhouses using artificial lighting such as LED. Furthermore, RGB distribution and micromole value could help improve the precision of SI-NDVI values that are affected by various light source spectrum, by implementing corrective coefficient [2] that is reflecting blue, red and far infrared distribution.

The article deals with development of a novel IoT based light sensor that can detect light distribution from semi-spherical direction, same time showing also RGB distribution, could improve precision of the lighting system switching ON/OFF algorithms, thus saving extra energy or provide more precise light amount to the plants, thus increasing the yield of crops.Application of RGB sensors that are able to obtain also energy values $\mathrm{W} \cdot \mathrm{m}^{-2}$, is promising, due to potential to decrease of the 
overall costs of such light sensor, as quantum sensors have the cost of 400-1000 EUR, the RGB sensor setup in the range of 100-200 EUR.

\section{The semi-spherical RGB sensor design}

If there is just one luminary (light source), the sensor development or placement is more comfortable. In essence, oriented into the direction of luminary reliable state RGB light sensor must be or concentrating light lens must be applied. If there are several luminaries, as well as many reflective surfaces, the measurement is very complicated and sometimes impossible - light at a point in space comes from surrounding 360 degrees.

A matrix of 14 RGB light sensors, positioned in the semi-spherical form (Fig. 1), allows estimating a level of light power and light spectrum at one point in the environment. Measured RGB data are sent wirelessly by WiFi or cellular network to the database, as well as to the light controller.

The proposed matrix consists of 14 RGB light sensors positioned in two circular vertical planes in the right angle to each other, and on each side of the circular plane, 3 or 4 sensors' printed circuit boards (PCB) are placed (Fig.1.). All sensors are enclosed into a transparent spherical bulb (ball) to protect the sensors' surface from dust and other particles. At the same time, plastic reduces the amount of light by 3-5\%. For precise measurements, a plastic enclosure (bulb) is not applied.
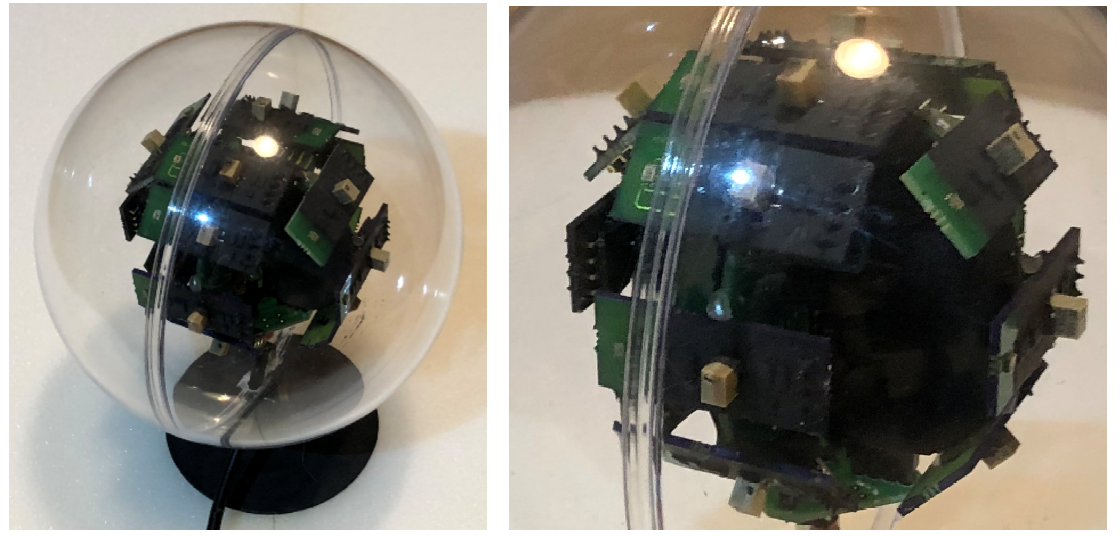

Fig. 1. RGB semi-spherical light sensor: overall image (left), closer look (right)

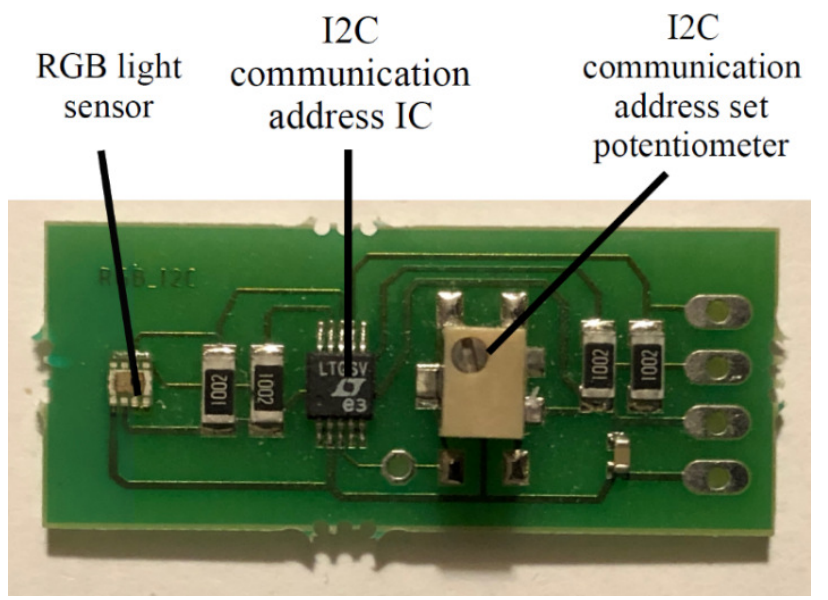

Fig. 2. Individual RGB light sensor

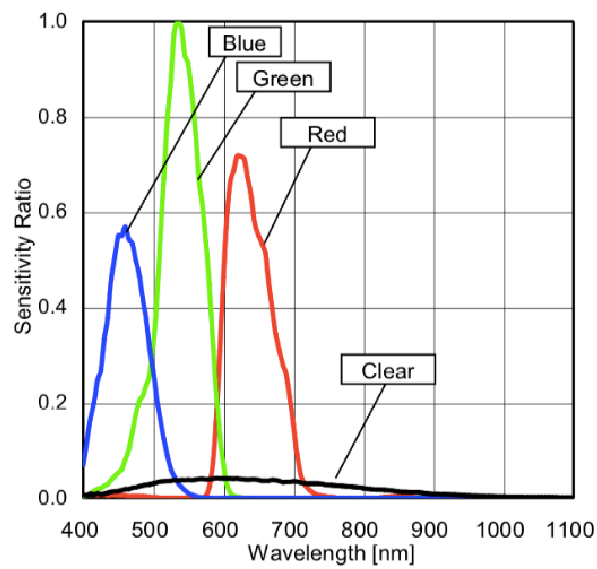

Fig. 3. Sensor sensitivity-wavelength relationship graph

Each RGB sensor printed PCB (Fig.2.) includes an RGB sensor (RhomBH1745NUC), I2C communication address set integrated circuit (IC), address set potentiometer, and additional elements. The RGB light sensor sensitivity - wavelength graph is shown in Fig.3. Sensor output allows calculating watt per square centimeter $\left(\mathrm{W} \cdot \mathrm{cm}^{-2}\right)$ at corresponding red $(\mathrm{R})$, green $(\mathrm{G})$, blue (B) wavelength, and overall "clear" received light value $(\mathrm{C})$. The obtained light of $\mathrm{R}, \mathrm{G}$ and $\mathrm{B}$ channels has an IR filter (passes visible light and blocks infrared light), but the clear " $\mathrm{C}$ " channel has no filter, thus it obtains light in full spectrum. Watt per square centimeter is the derived SI unit of illuminance 
and luminous emittance. Units $\mathrm{W} \cdot \mathrm{cm}^{-2}$ approximately can be recalculated to $\mu$ mols by the expression $[6]: 1 \mathrm{~W} \cdot \mathrm{m}^{-2} \approx 4.6 \mu$ mole $\cdot \mathrm{m}^{2} \cdot \mathrm{s}^{-1}$

The spherical view angle is approximately 30 spherical degrees in standard $3 \mathrm{~dB}$ sensitivity area for each sensor, measured at a right angle against the sensor surface. Sensor data output is a 16-bit digital value for each RGBC channel (covering 0-65535 decimal values). According to the data in Fig.4, it is possible to calculate the received $\mathrm{W} \cdot \mathrm{cm}^{-2}$. Fig. 4 values in the box represent the digital reading average value correspondence to $20 \mu \mathrm{W} \cdot \mathrm{cm}^{-2}$.

\begin{tabular}{|c|c|c|c|c|c|c|}
\hline Red Data Count Value & $D_{\text {RED }}$ & 3400 & 4000 & 4600 & count & $\begin{array}{l}\text { MODE_CONTROL } 2(42 \mathrm{~h})=12 \mathrm{~h}, \\
\mathrm{EV}=2 \overline{0} \mu \mathrm{W} / \mathrm{cm}^{2}\end{array}$ \\
\hline Green Data Count Value & D GREEN & 2847 & 3350 & 3853 & count & $\begin{array}{l}\text { MODE_CONTROL2 } 242 \mathrm{~h})=12 \mathrm{~h}, \\
\mathrm{EV}=20 \mu \mathrm{W} / \mathrm{cm}^{2}\end{array}$ \\
\hline Blue Data Count Value & $D_{\text {BLue }}$ & 2014 & 2370 & 2726 & count & $\begin{array}{l}\text { MODE_CONTROL2 } 2(42 \mathrm{~h})=12 \mathrm{~h}, \\
\mathrm{EV}=20 \overline{\mathrm{W}} / \mathrm{cm}^{2}\end{array}$ \\
\hline Clear Data Count Value & Dalear & 128 & 160 & 192 & count & $\begin{array}{l}\text { MODE_CONTROL2 } 2(42 h)=12 h \text {, } \\
E V=20 \mu W / \mathrm{cm}^{2}{ }_{(\text {Note }}^{4)}\end{array}$ \\
\hline Dark Count Value & $\mathrm{S}_{0 \_0}$ & 0 & 0 & 3 & count & $\begin{array}{l}\text { MODE_CONTROL2 }(42 h)=12 h \text {, } \\
\text { No input light }\end{array}$ \\
\hline
\end{tabular}

Fig. 4. Sensor output data for calculations according to sensor datasheet

Fig. 5. shows the sensors, marked as S0 to S13, positions on the spherical surface. Sensors S0, S1, S2, S3 and S7, S8, S9, S10, are located on one vertical plane but sensors S4, S5, S6 and S11, S12, S13 on another vertical plane. The planes are in a right angle to each other.

The overall data receiving and commands sending scheme are shown in Fig.6. Electric imp device [7] communicates with each sensor by the 2 -wire I2C protocol. The communication clock frequency is $10 \mathrm{kHz}$. Data are received sequentially from sensor to sensor.

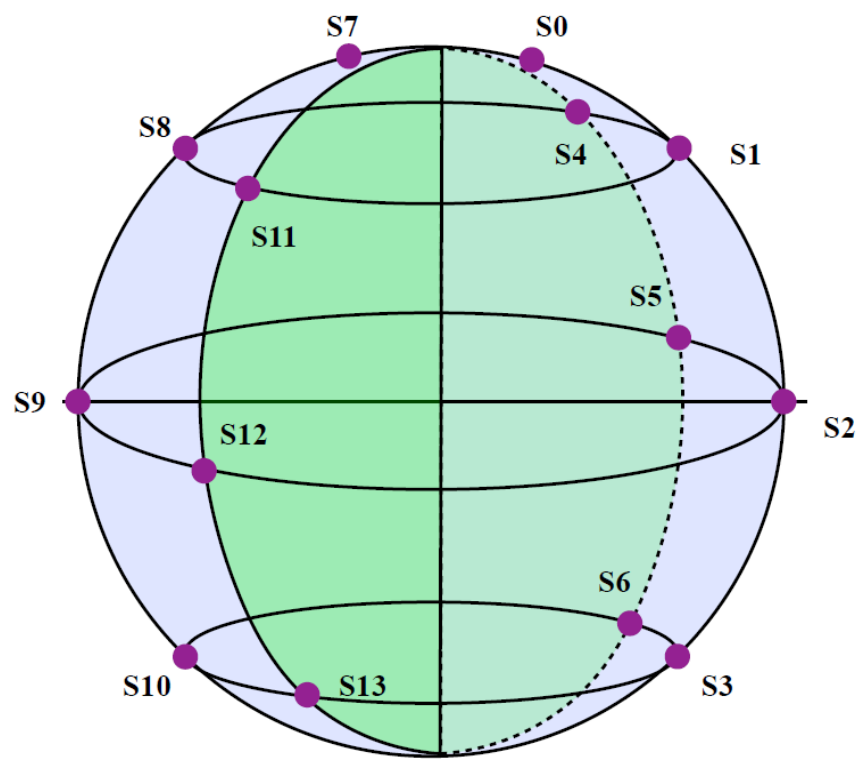

Fig. 5. Sensors' positions on the spherical surface

When data from all sensors are received, an electric imp device wirelessly by WiFi or cellular network sends them to a web server in CSV format where data are stored (see Fig. 6). Data processing to control the light system is an option. The data rate is set as 1 minute. This way we can obtain both instantaneous values and later also cumulative values can be obtained per day.

This article deals with the proof of concept evaluation of this sensor system, using rather simple testing method and environment. As the sensor is planned to be installed in the real greenhouse environment, then a cover must be applied to the sensor, to ensure appropriate IP rating (IP67 would be target), due to fact that real environment with high temperature and moisture values is very challenging for electronics. The cover will decrease the precision, but will prolong the lifetime of the sensor. At the moment tests will be performed for both cases -with cover and without cover, to see the 
impact of cover material to the results, and further also other types of materials will be tested in the same manner.

As next step of the research, testing in the controlled environment is planned, where 3 types of lighting will be used, same time also measurements from the spectrometer will be analysed, to compare the precision and enable calibration parameter calculation. Further the tests in industrial greenhouse will be performed to evaluate results of light parameters in real conditions, affected both by sunlight and greenhouse glass cover properties.

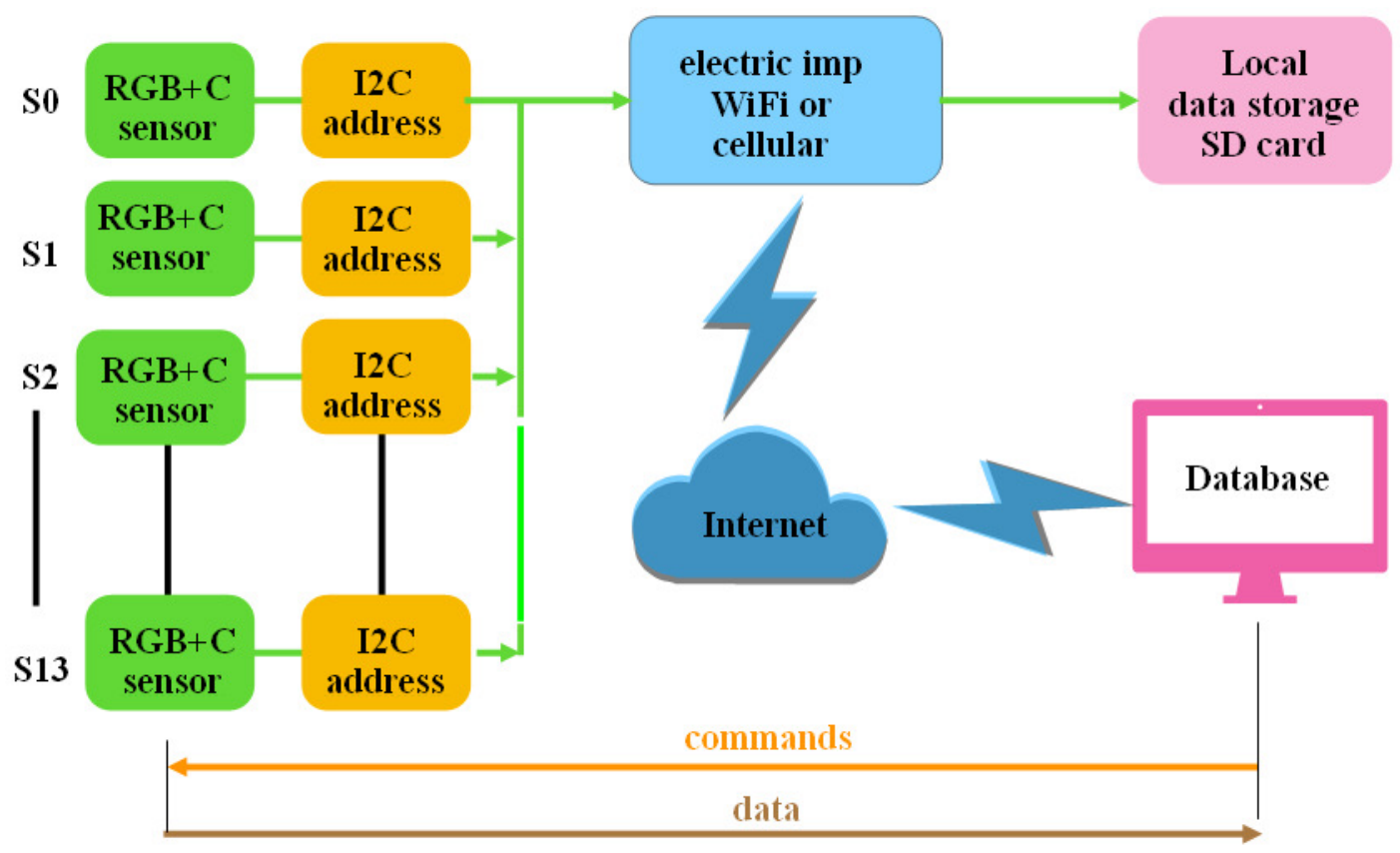

Fig. 6. Data receiving and commands sending scheme

In overall the RGB sensor system is similar to the described before systems [8; 9].

\section{Experimental results in the interpretation of data}

Each measurement contains 56 values -4 from each sensor multiplied by a count of sensors (14). This article deals with the main results from the first tests for proof of the concept. Due to a large number of table columns and rows, it is not possible to reflect all data here. Tests were provided in 4 conditions (Fig. 7). Table 1 includes raw data values from sensors S2, S5, S9, S12, for example. In all condition cases the natural light was used, using the same plant and room configuration.

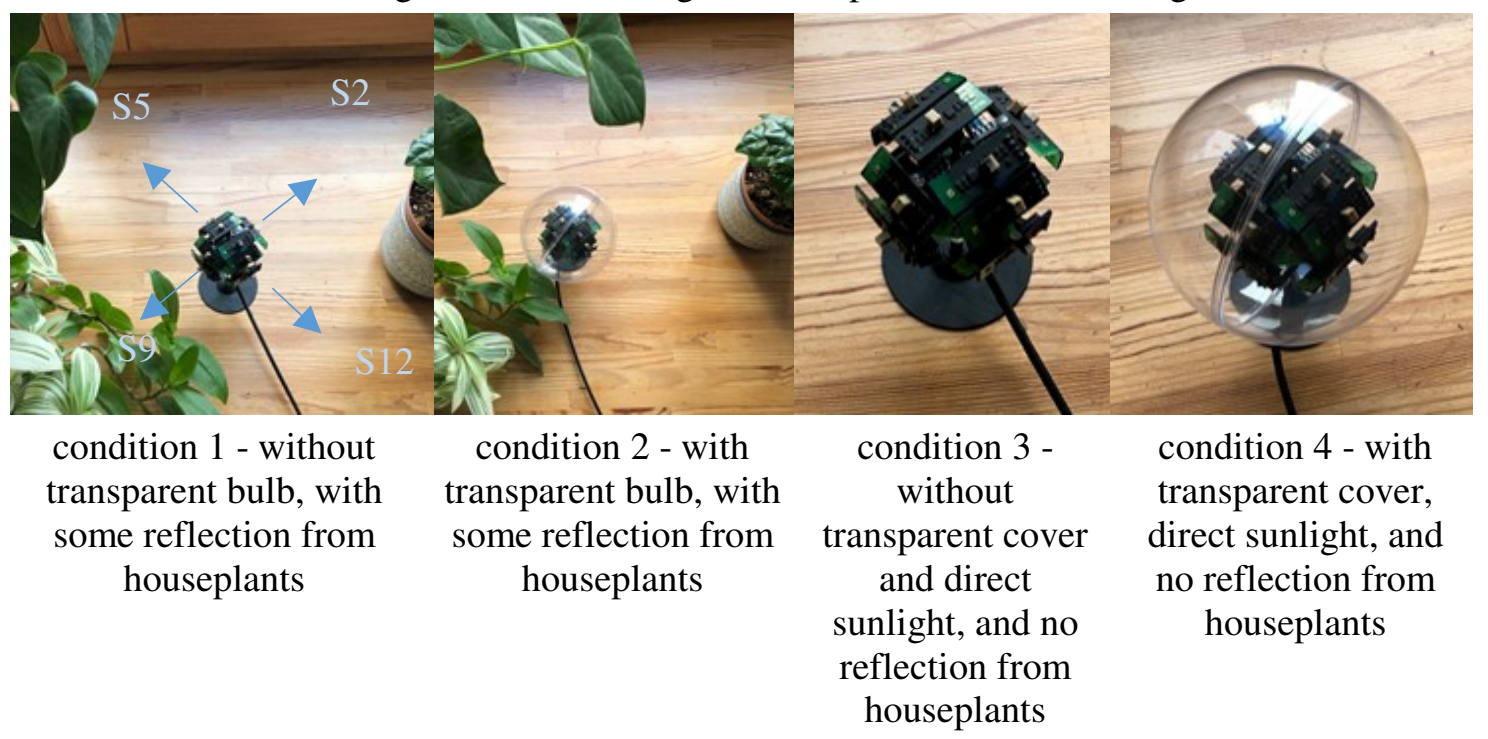

Fig. 7. Test conditions and description 
Raw data values from sensors S2, S5, S9, S12

Table 1

\begin{tabular}{|c|c|c|c|c|c|c|c|c|c|c|c|c|c|c|c|c|}
\hline Cond & R2 & G2 & B2 & C2 & R5 & G5 & B5 & C5 & R9 & G9 & B9 & C9 & R12 & G12 & B12 & C12 \\
\hline 1 & 2786 & 8899 & 3763 & 1793 & 3283 & 10027 & 4409 & 1900 & 11 & 693 & 183 & 242 & 87 & 1266 & 351 & 433 \\
\hline 2 & 2232 & 7628 & 3122 & 1580 & 3543 & 9652 & 4148 & 1842 & 126 & 798 & 232 & 259 & 35 & 1215 & 338 & 404 \\
\hline 3 & 3237 & 10026 & 4311 & 1955 & 3633 & 10482 & 4584 & 1984 & 235 & 973 & 268 & 308 & 609 & 1693 & 542 & 511 \\
\hline 4 & 2794 & 8649 & 3665 & 1725 & 3786 & 9927 & 4264 & 1895 & 279 & 1379 & 429 & 327 & 603 & 1711 & 565 & 498 \\
\hline
\end{tabular}

In order to calculate each channel energy value, the corresponding coefficient (given at $20 \mu \mathrm{W} \cdot \mathrm{cm}^{-2}$, see Fig.4) must be used. Further the radiant energy value can be multiplied by 4.57 (for sunlight) to obtain the PAR $\left(\mu\right.$ mole $\left.\cdot \mathrm{m}^{2} \cdot \mathrm{s}^{-1}\right)$ value.

Calculated PPFD values from sensors S2, S5, S9, S12

Table 2

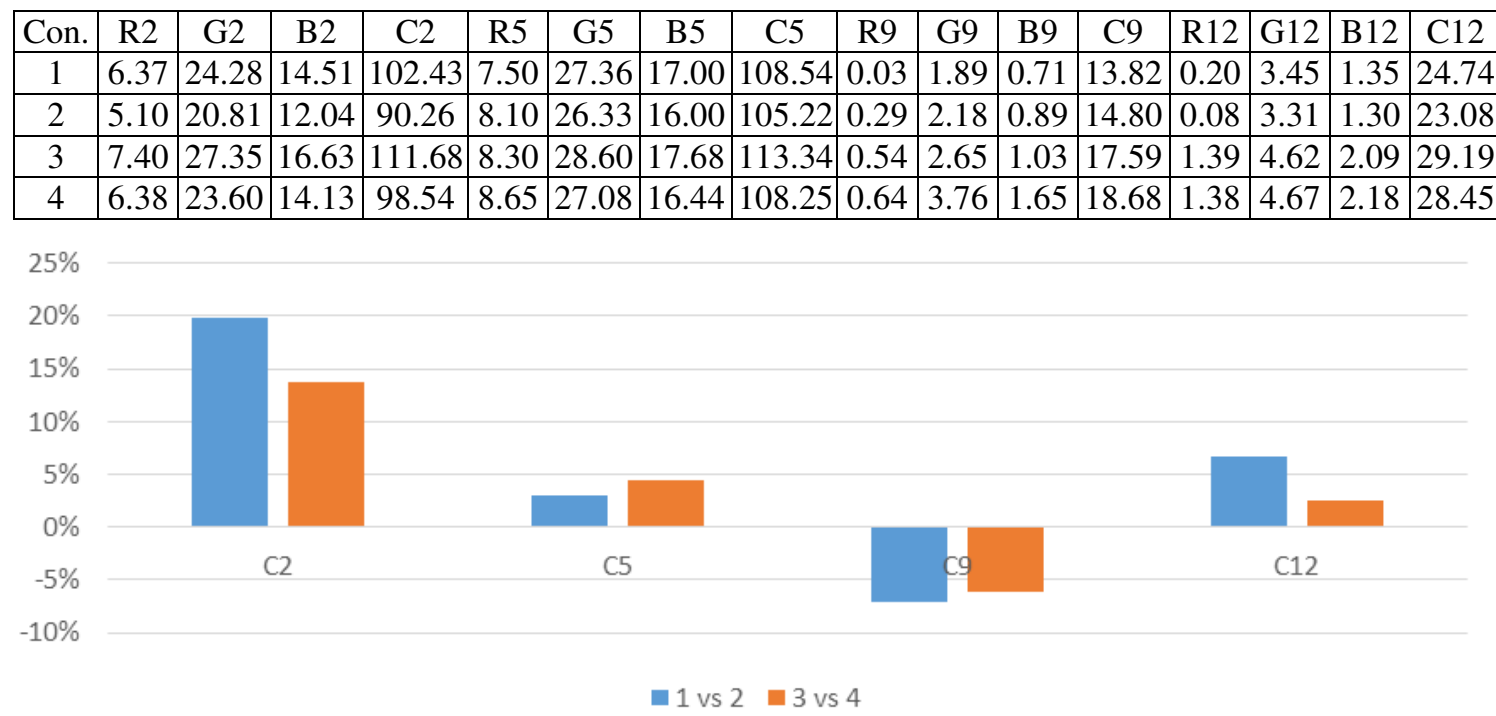

Fig. 8. Difference between conditions $1 / 2$ and $3 / 4$ for "clear" channels

In Fig. 8, we can observe that there is impact of the cover, ranging from $3 \%$ to $20 \%$ for condition 1-2, and 3-14\% for condition 3-4 comparison. Also, the results show that with more light, also the readings are with less difference. The values also show from which side the most of light came (S2 and S5), corresponding to the placement towards the sunlight. S9 values have the least values, as the least amount of light was received, probably only the reflected light.

For further analysis, also $\mathrm{R}+\mathrm{G}+\mathrm{B}$ and/or $\mathrm{C}$ values should be compared, as it would be a good indicator for light distribution (or luminary type) ratio detection, that can be used further in SI-NDVI sensors.

\section{Conclusions}

1. Light measurements typically reflect some luminaire emitting light and illumination on known distance or in sphere [10].

2. The article deals with another look - receiving the light (and light RGB components) on the spot from the surrounding $360^{\circ}$ environments and a proof of the concept for such measurement device.

3. The described $360^{\circ}$ spherically placed sensor matrix shows good results for each sensor, but the overall received light power calculation method is still on the discussion. The work continues.

4. The received data rate from sensors can be up to $1 \mathrm{sec}$, and such rate can reflect received light changes very effectively.

\section{Acknowledgements}

The publication is created with support of the Latvian Rural Development Program 2014-2020 "Cooperation", called 16.1 project No. 19-00-A01612-000010 "Investigation of innovative solutions and new method development for efficiency and quality increase in Latvian greenhouse sector" [IRIS]. 


\section{References}

[1] Li-COR Quantum PAR sensor. [online][11.02.2020] Available at: https://www.licor.com/env/products/light/

[2] Avotins A, Kviesis K, Bicans J., Alsina I. Dubova L. Experimental analysis of IoT based camera SI-NDVI values for tomato plant health monitoring application. Proceedings of 11th International conference on Biosystems Engineering, BSE2020, May 6-8, 2020, Tartu, Estonia. (in press)

[3] Botha E.J. Estimating nitrogen status on crops using non destructing remote sensing technique. Republic of South Africa. Academic Press. Inc., 2001, pp. 1-10.

[4] Katsoulas N., Elvanidi A., Ferentinos K.P., Kacira M., Bartzanas T., Kittas C., Crop reflectance monitoring as a tool for water stress detection in greenhouses: A review, Biosystems Engineering, Volume 151, 2016, pp. 374-398.

[5] Nicole S. Beisel, Jordan B. Callaham, Natasha J. Sng, Dylan J. Taylor, Anna-Lisa Paul, Robert J. Ferl. Utilization of single-image normalized difference vegetation index (SINDVI) for early plant stress detection. Applications in Plant Sciences, 2018; 6 (10).

[6] Sager J., Farlane C., Plant Growth Chamber Handbook, Chapter 1- Radiation, [online][11.02.2020] Available at: https://www.controlledenvironments.org/wpcontent/uploads/sites/6/2017/06/Ch01.pdf

[7] Description of Electric imp device. [online][11.02.2020] Available at: www.electricimp.com

[8] Apse-Apsitis P., Avotins A., Porins R., Industrial greenhouse electrical power monitoring using secure internet-of-things(IoT) Platform, 2018 IEEE 6th Workshop on Advances in Information, Electronic and Electrical Engineering, AIEEE 2018 - Proceedings.

[9] Avotins A., Apse-Apsitis P., Bicans J., Gruduls J., Development and testing results of IoT based air temperature and humidity measurement system for industrial greenhouse, Agronomy Research, Volume 16, Issue Special Issue 1, 2018, pp. 943-951.

[10] Ryer A., Light measurement Handbook, International Light, Newburyport, MA, ISBN 09658356-9-3, [online][11.02.2020] Available at: http://www.dfisica.ubi.pt/ hgil/FotoMetria/HandBook/Lensbig.gif 